\title{
GENERALIZED STRONGLY SET-VALUED NONLINEAR COMPLEMENTARITY PROBLEMS
}

\author{
NAN-JING HUANG and YEOL JE CHO
}

(Received 7 October 1997)

\begin{abstract}
In this paper, we introduce a new class of generalized strongly set-valued nonlinear complementarity problems and construct new iterative algorithms. We show the existence of solutions for this kind of nonlinear complementarity problems and the convergence of iterative sequences generated by the algorithm. Our results extend some recent results in this field.

Keywords and phrases. Complementarity problem, strongly monotone and Lipschitz continuous mappings, strongly monotone and $H$-Lipschitz continuous set-valued mappings, algorithm.
\end{abstract}

1991 Mathematics Subject Classification. 90C33, 47H04.

1. Introduction. The complementarity theory, which was introduced by Lemke [11], Cottle, and Dantzig [6] in the early 1960s and later developed by others, plays an important and fundamental role in the study of a wide class of problems arising in mechanics, physics, control and optimization, economics and transportation equilibrium, contact problems in elasticity, fluid flow through porous media, and many other branches of mathematical and engineering sciences [1, 2, 3, 4, 5, 7, 8, 9, 10, 13, 14, 15]. In particular, the set-valued quasi-(implicit)complementarity problems, considered and studied by Chang and Huang [2, 3], are important among the generalizations of the complementarity problems. In [14], Noor introduced and studied some new classes of nonlinear complementarity problems for single-valued mappings in $\mathbb{R}^{n}$ and, in [4], Chang and Huang introduced and studied some new nonlinear complementarity problems for compact-valued fuzzy mappings and set-valued mappings which include many kinds of complementarity problems, considered by Chang [1], Cottle et al. [7], Isac [9], and Noor [13, 14], as special cases.

In this paper, we introduce and study a new class of generalized strongly set-valued nonlinear complementarity problems and construct new iterative algorithms. We also discuss the existence of solutions for this kind of nonlinear complementarity problems and the convergence of iterative sequences generated by the algorithm. Our results improve and develop some results in [4, 13, 14].

2. Preliminaries. Let $\mathbb{R}^{n}$ be the Euclidean space endowed with norm $\|\cdot\|$ and inner product $(\cdot, \cdot)$, respectively. In the sequel, we use the following notations:

$$
\begin{gathered}
2^{\mathbb{R}^{n}}=\left\{A: A \subset \mathbb{R}^{n} \text { and } A \text { is nonempty }\right\}, \\
C B\left(\mathbb{R}^{n}\right)=\left\{A: A \subset \mathbb{R}^{n} \text { and } A \text { is nonempty, bounded, and closed }\right\},
\end{gathered}
$$




$$
|x|=\left(\left|x_{1}\right|,\left|x_{2}\right|, \ldots,\left|x_{n}\right|\right) \quad \text { for all } x \in \mathbb{R}^{n} \text {. }
$$

Let $F, G, Q: \mathbb{R}^{n} \rightarrow 2^{\mathbb{R}^{n}}$ be three set-valued mappings and $f, g: \mathbb{R}^{n} \rightarrow \mathbb{R}^{n}$ be two single-valued mappings. Now, we consider the following problem.

Find $u \in \mathbb{R}^{n}, x \in F(u), y \in G(u)$, and $z \in Q(y)$ such that

$$
u \geq 0, \quad f(x)+g(z) \geq 0, \quad(u, f(x)+g(x))=0 .
$$

This problem is called the generalized strongly set-valued nonlinear complementarity problem.

If $Q$ is the identity mapping on $\mathbb{R}^{n}$, then problem (2.4) is equivalent to the following. Find $u \in \mathbb{R}^{n}, x \in F(u)$ and $y \in G(u)$ such that

$$
u \geq 0, \quad f(x)+g(y) \geq 0, \quad(u, f(x)+g(y))=0 .
$$

This problem is called the generalized set-valued nonlinear complementarity problem.

If $F$ is the identity mapping on $\mathbb{R}^{n}$, then problem (2.5) is equivalent to the following. Find $u \in \mathbb{R}^{n}$ and $y \in G(u)$ such that

$$
u \geq 0, \quad f(u)+g(y) \geq 0, \quad(u, f(u)+g(y))=0,
$$

which was considered by Chang and Huang in [4].

If $F=G$ is the identity mapping on $\mathbb{R}^{n}$, then problem (2.5) is equivalent to the following.

Find $u \in \mathbb{R}^{n}$ such that

$$
u \geq 0, \quad f(u)+g(u) \geq 0, \quad(u, f(u)+g(u))=0,
$$

which was considered by Noor [14].

If $F=G$ is the identity mapping on $\mathbb{R}^{n}$ and $f \equiv 0$, then problem (2.5) is equivalent to the following.

Find $u \in \mathbb{R}^{n}$ such that

$$
u \geq 0, \quad g(u) \geq 0, \quad(u, g(u))=0,
$$

which was considered by Karamardian [10], Fang [8], and Noor [13].

Obviously, problem (2.4) can be written as follows:

Find $u \in \mathbb{R}^{n}, x \in F(u), y \in G(u)$ and $z \in Q(y)$ such that

$$
u \geq 0, \quad v=f(x)+g(z) \geq 0, \quad(u, v)=0 .
$$

We now consider the following equalities:

$$
u=\frac{1}{2}(|w|+w), \quad v=(\lambda \rho)^{-1}(|w|-w),
$$

where $\lambda, \rho>0$ are constants. Clearly, $u \geq 0$ and $v \geq 0$. From (2.5) and (2.9), it follows that problem (2.9) is equivalent to the following.

Find $w \in \mathbb{R}^{n}, x \in F(1 / 2(|w|+w)), y \in G(1 / 2(|w|+w))$, and $z \in Q(y)$ such that

$$
w=\frac{1}{2}(|w|+w)-\frac{1}{2} \lambda \rho(f(x)+g(z)),
$$

where $\lambda, \rho>0$ are constants. 
3. Algorithms. Based on the formulations in Section 2, we now construct the new algorithms for the generalized strongly set-valued nonlinear complementarity problem (2.4).

Let $F, G, Q: \mathbb{R}^{n} \rightarrow \mathrm{CB}\left(\mathbb{R}^{n}\right)$ be three set-valued mappings and let $f, g: \mathbb{R}^{n} \rightarrow \mathbb{R}^{n}$ be two single-valued mappings. For any $w_{o} \in \mathbb{R}^{n}$, let

$$
x_{0} \in F\left(\frac{1}{2}\left(\left|w_{0}\right|+w_{0}\right)\right), \quad y_{0} \in G\left(\frac{1}{2}\left(\left|w_{0}\right|+w_{0}\right)\right), \quad z_{0} \in Q\left(y_{0}\right)
$$

and

$$
w_{1}=\frac{1}{2}(1-\lambda)\left(\left|w_{0}\right|+w_{0}\right)+\frac{1}{2} \lambda\left(\left|w_{0}\right|+w_{0}-\rho\left(f\left(x_{0}\right)+g\left(z_{0}\right)\right)\right),
$$

where $\lambda, \rho>0$ are constants.

Since $x_{0} \in F\left(1 / 2\left(\left|w_{0}\right|+w_{0}\right)\right), y_{0} \in G\left(1 / 2\left(\left|w_{0}\right|+w_{0}\right)\right)$ and $z_{0} \in Q\left(y_{0}\right)$, by Nadler [12], there exist $x_{1} \in F\left(1 / 2\left(\left|w_{1}\right|+w_{1}\right)\right), y_{1} \in G\left(1 / 2\left(\left|w_{1}\right|+w_{1}\right)\right)$ and $z_{1} \in Q\left(y_{1}\right)$ such that

$$
\begin{aligned}
& \left\|x_{0}-x_{1}\right\| \leq(1+1) H\left(F\left(\frac{1}{2}\left(\left|w_{0}\right|+w_{0}\right)\right), F\left(\frac{1}{2}\left(\left|w_{1}\right|+w_{1}\right)\right)\right), \\
& \left\|y_{0}-y_{1}\right\| \leq(1+1) H\left(G\left(\frac{1}{2}\left(\left|w_{0}\right|+w_{0}\right)\right), G\left(\frac{1}{2}\left(\left|w_{1}\right|+w_{1}\right)\right)\right), \\
& \left\|z_{1}-z_{0}\right\| \leq(1+1) H\left(Q\left(y_{1}\right), Q\left(y_{0}\right)\right),
\end{aligned}
$$

where $H(\cdot, \cdot)$ denotes the Hausdorff metric on $\mathrm{CB}\left(\mathbb{R}^{n}\right)$.

Thus, by induction, we can obtain the following algorithm:

Algorithm 3.1. Let $F, G, Q: \mathbb{R}^{n} \rightarrow \mathrm{CB}\left(\mathbb{R}^{n}\right)$ be three set-valued mappings and let $f, g: \mathbb{R}^{n} \rightarrow \mathbb{R}^{n}$ be two single-valued mappings. For any $w_{0} \in \mathbb{R}^{n}$, we can construct sequences $\left\{w_{n}\right\},\left\{x_{n}\right\},\left\{y_{n}\right\}$, and $\left\{z_{n}\right\}$ in $\mathbb{R}^{n}$ as follows:

$$
\begin{aligned}
& x_{n} \in F\left(\frac{1}{2}\left(\left|w_{n}\right|+w_{n}\right)\right), \quad y_{n} \in G\left(\frac{1}{2}\left(\left|w_{n}\right|+w_{n}\right)\right), \quad z_{n} \in Q\left(y_{n}\right), \\
& \left\|x_{n}-x_{n+1}\right\| \leq\left(1+\frac{1}{n+1}\right) H\left(F\left(\frac{1}{2}\left(\left|w_{n}\right|+w_{n}\right)\right), F\left(\frac{1}{2}\left(\left|w_{n+1}\right|+w_{n+1}\right)\right)\right), \\
& \left\|y_{n}-y_{n+1}\right\| \leq\left(1+\frac{1}{n+1}\right) H\left(G\left(\frac{1}{2}\left(\left|w_{n}\right|+w_{n}\right)\right), G\left(\frac{1}{2}\left(\left|w_{n+1}\right|+w_{n+1}\right)\right)\right), \\
& \left\|z_{n}-z_{n+1}\right\| \leq\left(1+\frac{1}{n+1}\right) H\left(Q\left(y_{n}\right), Q\left(y_{n+1}\right)\right), \\
& w_{n+1}=\frac{1}{2}(1-\lambda)\left(\left|w_{n}\right|+w_{n}\right)+\frac{1}{2} \lambda\left(\left|w_{n}\right|+w_{n}-\rho\left(f\left(x_{n}\right)+g\left(z_{n}\right)\right)\right)
\end{aligned}
$$

for $n=0,1,2, \ldots$, where $\lambda, \rho>0$ are constants.

From Algorithm 3.1, we can obtain the following algorithms.

Algorithm 3.2. Let $F, G: \mathbb{R}^{n} \rightarrow \mathrm{CB}\left(\mathbb{R}^{n}\right)$ be two set-valued mappings and let $f, g$ : $\mathbb{R}^{n} \rightarrow \mathbb{R}^{n}$ be two single-valued mappings. For any $w_{0} \in \mathbb{R}^{n}$, we can construct sequences 
$\left\{w_{n}\right\},\left\{x_{n}\right\}$, and $\left\{y_{n}\right\}$ in $\mathbb{R}^{n}$ as follows:

$$
\begin{aligned}
& x_{n} \in F\left(\frac{1}{2}\left(\left|w_{n}\right|+w_{n}\right)\right), \quad y_{n} \in G\left(\frac{1}{2}\left(\left|w_{n}\right|+w_{n}\right)\right), \\
& \left\|x_{n}-x_{n+1}\right\| \leq\left(1+\frac{1}{n+1}\right) H\left(F\left(\frac{1}{2}\left(\left|w_{n}\right|+w_{n}\right)\right), F\left(\frac{1}{2}\left(\left|w_{n+1}\right|+w_{n+1}\right)\right)\right), \\
& \left\|y_{n}-y_{n+1}\right\| \leq\left(1+\frac{1}{n+1}\right) H\left(G\left(\frac{1}{2}\left(\left|w_{n}\right|+w_{n}\right)\right), G\left(\frac{1}{2}\left(\left|w_{n+1}\right|+w_{n+1}\right)\right)\right), \\
& w_{n+1}=\frac{1}{2}(1-\lambda)\left(\left|w_{n}\right|+w_{n}\right)+\frac{1}{2} \lambda\left(\left|w_{n}\right|+w_{n}-\rho\left(f\left(x_{n}\right)+g\left(z_{n}\right)\right)\right)
\end{aligned}
$$

for $n=0,1,2, \ldots$, where $\lambda, \rho>0$ are constants.

Algorithm 3.3 [4]. Let $G: \mathbb{R}^{n} \rightarrow \mathrm{CB}\left(\mathbb{R}^{n}\right)$ be a set-valued mapping and let $f, g$ : $\mathbb{R}^{n} \rightarrow \mathbb{R}^{n}$ be two single-valued mappings. For any $w_{0} \in \mathbb{R}^{n}$, we can construct sequences $\left\{w_{n}\right\}$ and $\left\{y_{n}\right\}$ in $\mathbb{R}^{n}$ as follows:

$$
\begin{aligned}
& y_{n} \in G\left(\frac{1}{2}\left(\left|w_{n}\right|+w_{n}\right)\right), \\
& \| y_{n}-y_{n+1}|| \leq\left(1+\frac{1}{n+1}\right) H\left(G\left(\frac{1}{2}\left(\left|w_{n}\right|+w_{n}\right)\right), G\left(\frac{1}{2}\left(\left|w_{n+1}\right|+w_{n+1}\right)\right)\right), \\
& w_{n+1}=\frac{1}{2}(1-\lambda)\left(\left|w_{n}\right|+w_{n}\right)+\frac{1}{2} \lambda\left(\left|w_{n}\right|+w_{n}-\rho\left(f\left(\frac{1}{2}\left(\left|w_{n}\right|+w_{n}\right)\right)+g\left(y_{n}\right)\right)\right)
\end{aligned}
$$

for $n=0,1,2, \ldots$, where $\lambda, \rho>0$ are constants.

Algorithm 3.4. [14] Let $f, g: \mathbb{R}^{n} \rightarrow \mathbb{R}^{n}$ be two single-valued mappings. For any $w_{0} \in \mathbb{R}^{n}$, we can construct sequences $\left\{w_{n}\right\}$ and $\left\{y_{n}\right\}$ in $\mathbb{R}^{n}$ as follows.

$$
\begin{aligned}
y_{n}= & \frac{1}{2}\left(\left|w_{n}\right|+w_{n}\right), \\
w_{n+1}= & \frac{1}{2}(1-\lambda)\left(\left|w_{n}\right|+w_{n}\right) \\
& +\frac{1}{2} \lambda\left(\left|w_{n}\right|+w_{n}-\rho\left(f\left(\frac{1}{2}\left(\left|w_{n}\right|+w_{n}\right)\right)+g\left(y_{n}\right)\right)\right)
\end{aligned}
$$

for $n=0,1,2, \ldots$, where $\lambda, \rho>0$ are constants.

Algorithm 3.5. [15] Let $g: \mathbb{R}^{n} \rightarrow \mathbb{R}^{n}$ be a single-valued mapping. For any $w_{0} \in \mathbb{R}^{n}$, we can construct sequences $\left\{w_{n}\right\}$ and $\left\{y_{n}\right\}$ in $\mathbb{R}^{n}$ as follows.

$$
\begin{aligned}
y_{n} & =\frac{1}{2}\left(\left|w_{n}\right|+w_{n}\right), \\
w_{n+1} & =\frac{1}{2}(1-\lambda)\left(\left|w_{n}\right|+w_{n}\right)+\frac{1}{2} \lambda\left(\left|w_{n}\right|+w_{n}-\rho\left(g\left(y_{n}\right)\right)\right)
\end{aligned}
$$

for $n=0,1,2, \ldots$, where $\lambda, \rho>0$ are constants.

4. Existence and convergence. In this section, we show the existence of solutions for the generalized strongly set-valued nonlinear complementarity problem (2.4) and the convergence of the iterative sequences constructed by Algorithm 3.1. We first give some definitions.

\section{DEFINITIONS 4.1.}

(1) A mapping $f: \mathbb{R}^{n} \rightarrow \mathbb{R}^{n}$ is said to be strongly monotone if there exists a constant $\alpha>0$ such that

$$
(f(u)-f(v), u-v) \geq \alpha\|u-v\|^{2}
$$


for all $u, v \in \mathbb{R}^{n}$.

(2) A mapping $f: \mathbb{R}^{n} \rightarrow \mathbb{R}^{n}$ is said to be Lipschitz continuous if there exists a constant $\beta>0$ such that

$$
\|f(u)-f(v)\| \leq \beta\|u-v\|
$$

for all $u, v \in \mathbb{R}^{n}$. The number $\beta$ in (2) is called Lipschitz constant.

It is easy to see that $\alpha \leq \beta$.

\section{DEFINITIONS 4.2.}

(1) A set-valued mapping $F: \mathbb{R}^{n} \rightarrow \mathrm{CB}\left(\mathbb{R}^{n}\right)$ is said to be strongly monotone with respect to the mapping $f: \mathbb{R}^{n} \rightarrow \mathbb{R}^{n}$ if there exists a constant $\alpha>0$ such that

$$
(f(x)-f(y), u-v) \geq \alpha\|u-v\|^{2}
$$

for all $u, v \in \mathbb{R}^{n}, x \in F(u)$ and $y \in F(v)$.

(2) A set-valued mapping $F: \mathbb{R}^{n} \rightarrow \mathrm{CB}\left(\mathbb{R}^{n}\right)$ is said to be H-Lipschitz continuous if there exists a constant $\beta>0$ such that

$$
H(F(u), F(v)) \leq \beta\|u-v\|
$$

for all $u, v \in \mathbb{R}^{n}$. The number $\beta$ in (2) is called the $H$-Lipschitz constant.

Now, we give our main theorems in this paper.

Theorem 4.1. Suppose that $f, g: \mathbb{R}^{n} \rightarrow \mathbb{R}^{n}$ are Lipschitz continuous with Lipschitz constants $\delta$ and $\xi$, respectively, and $F, G, Q: \mathbb{R}^{n} \rightarrow \mathrm{CB}\left(\mathbb{R}^{n}\right)$ are H-Lipschitz continuous with $H$-Lipschitz constants $\beta, \eta, v$, respectively, and $F$ is strongly monotone with respect to $f$ with strongly monotone constant $\alpha$. If

$$
0<\rho<\frac{4(\alpha-\xi \nu \eta)}{(\delta \beta)^{2}-(\xi \nu \eta)^{2}}, \quad \rho \xi \nu \eta<2, \quad \xi \nu \eta<\min \{\alpha, \delta \beta\},
$$

then there exist $u, x, y, z \in \mathbb{R}^{n}$ which solve problem (2.4). Furthermore, it follows that

$$
\frac{1}{2}\left(\left|w_{n}\right|+w_{n}\right) \rightarrow u, \quad x_{n} \rightarrow x, \quad y_{n} \rightarrow y, \quad z_{n} \rightarrow z \quad \text { as } n \rightarrow \infty,
$$

where $\left\{w_{n}\right\},\left\{x_{n}\right\},\left\{y_{n}\right\}$, and $\left\{z_{n}\right\}$ in $\mathbb{R}^{n}$ are the sequences generated by Algorithm 3.1.

Proof. By Algorithm 3.1, we have

$$
\begin{aligned}
\| w_{n+1}- & w_{n} \| \\
= & \| \frac{1}{2}(1-\lambda)\left(\left|w_{n}\right|+w_{n}\right)+\frac{1}{2} \lambda\left(\left|w_{n}\right|+w_{n}-\rho\left(f\left(x_{n}\right)+g\left(z_{n}\right)\right)\right) \\
& \quad-\frac{1}{2}(1-\lambda)\left(\left|w_{n-1}\right|+w_{n-1}\right)-\frac{1}{2} \lambda\left(\left|w_{n-1}\right|+w_{n-1}-\rho\left(f\left(x_{n-1}\right)+g\left(z_{n-1}\right)\right)\right) \| \\
\leq & (1-\lambda)\left\|w_{n}-w_{n-1}||+\frac{1}{2} \lambda \rho\right\| g\left(z_{n}\right)-g\left(z_{n-1}\right) \| \\
& +\lambda\left\|\frac{1}{2}\left(\left|w_{n}\right|+w_{n}\right)-\frac{1}{2}\left(\left|w_{n-1}\right|+w_{n-1}\right)-\frac{1}{2} \rho\left(f\left(x_{n}\right)-f\left(x_{n-1}\right)\right)\right\| .
\end{aligned}
$$


Since $F, G$, and $Q$ are $H$-Lipschitz continuous and $f$ and $g$ are Lipschitz continuous, from (3.6), it follows that

$$
\begin{aligned}
\left\|f\left(x_{n}\right)-f\left(x_{n-1}\right)\right\| & \leq \delta\left\|x_{n}-x_{n-1}\right\| \\
& \leq \delta\left(1+\frac{1}{n}\right) H\left(F\left(\frac{1}{2}\left(\left|w_{n}\right|+w_{n}\right)\right), F\left(\frac{1}{2}\left(\left|w_{n-1}\right|+w_{n-1}\right)\right)\right) \\
& \leq \delta\left(1+\frac{1}{n}\right) \beta\left\|\frac{\left|w_{n}\right|+w_{n}}{2}-\frac{\left|w_{n-1}\right|+w_{n-1}}{2}\right\| \\
& \leq \delta\left(1+\frac{1}{n}\right) \beta\left\|w_{n}-w_{n-1}\right\|
\end{aligned}
$$

and

$$
\begin{aligned}
\| g\left(z_{n}\right) & -g\left(z_{n-1}\right) \| \\
& \leq \xi\left\|z_{n}-z_{n-1}\right\| \leq \xi\left(1+\frac{1}{n}\right) H\left(Q\left(y_{n}\right), Q\left(y_{n-1}\right)\right) \\
& \leq \xi v\left(1+\frac{1}{n}\right)\left\|y_{n}-y_{n-1}\right\| \\
& \leq \xi v\left(1+\frac{1}{n}\right)^{2} H\left(G\left(\left(\frac{1}{2}\left(\left|w_{n}\right|+w_{n}\right)\right), G\left(\frac{1}{2}\left(\left|w_{n-1}\right|+w_{n-1}\right)\right)\right)\right) \\
& \leq \xi v\left(1+\frac{1}{n}\right)^{2} \eta\left\|w_{n}-w_{n-1}\right\| .
\end{aligned}
$$

Further, from the strong monotonicity of $F$ with respect to $f$ and (4.8), we have

$$
\begin{aligned}
\| \frac{1}{2}\left(\left|w_{n}\right|+w_{n}\right) & -\frac{1}{2}\left(\left|w_{n-1}\right|+w_{n-1}\right)-\frac{1}{2} \rho\left(f\left(x_{n}\right)-f\left(x_{n-1}\right)\right) \|^{2} \\
& \leq\left(1-\alpha \rho+\frac{1}{4} \rho^{2} \delta^{2}\left(1+\frac{1}{n}\right)^{2} \beta^{2}\right)\left\|w_{n}-w_{n-1}\right\|^{2} .
\end{aligned}
$$

Thus, it follows, from (4.7), (4.8), (4.9), and (4.10), that

$$
\begin{aligned}
\| w_{n+1} & -w_{n} \| \\
& \leq\left(1-\lambda+\frac{1}{2} \lambda \rho \xi \eta\left(1+\frac{1}{n}\right)+\lambda \sqrt{1-\alpha \rho+\frac{1}{4} \rho^{2} \delta^{2} \beta^{2}\left(1+\frac{1}{n}\right)^{2}}\right)\left\|w_{n}-w_{n-1}\right\| \\
& =\theta_{n}\left\|w_{n}-w_{n-1}\right\|,
\end{aligned}
$$

where

$$
\theta_{n}=1-\lambda+\frac{1}{2} \lambda \rho \xi \nu \eta\left(1+\frac{1}{n}\right)^{2}+\lambda \sqrt{1-\alpha \rho+\frac{1}{4} \rho^{2} \delta^{2} \beta^{2}\left(1+\frac{1}{n}\right)^{2}} .
$$

Letting

$$
\theta=1-\lambda+\frac{1}{2} \lambda \rho \xi \nu \eta+\lambda \sqrt{1-\alpha \rho+\frac{1}{4} \rho^{2} \delta^{2} \beta^{2}},
$$

$\theta_{n} \rightarrow \theta$ as $n \rightarrow \infty$. In view of (4.5), we know that $0<\theta<1$ and so $\theta_{n}<1$ for $n$ sufficiently large. It follows from (4.11) that $\left\{w_{n}\right\}$ is a Cauchy sequence in $\mathbb{R}^{n}$ and so 
we can suppose that $w_{n} \rightarrow w$ as $n \rightarrow \infty$. (4.8) and (4.9) imply that $\left\{x_{n}\right\},\left\{y_{n}\right\}$, and $\left\{z_{n}\right\}$ are also Cauchy sequences in $\mathbb{R}^{n}$. Let $x_{n} \rightarrow x, y_{n} \rightarrow y$, and $z_{n} \rightarrow z$ as $n \rightarrow \infty$. Letting $u=\frac{1}{2}(|w|+w)$, we get

$$
\frac{1}{2}\left(\left|w_{n}\right|+w_{n}\right) \rightarrow u, \quad x_{n} \rightarrow x, \quad y_{n} \rightarrow y, \quad z_{n} \rightarrow z \quad \text { as } n \rightarrow \infty .
$$

Now, we prove that $x \in F(u), y \in G(u)$, and $z \in Q(y)$. In fact, we have

$$
\begin{aligned}
d(x, F(u)) & =\inf \{\|x-z\|: z \in F(u)\} \\
& \leq\left\|x-x_{n}\right\|+d\left(x_{n}, F(u)\right) \\
& \leq\left\|x-x_{n}\right\|+H\left(F\left(\frac{1}{2}\left(\left|w_{n}\right|+w_{n}\right)\right), F(u)\right) \\
& \leq\left\|x-x_{n}\right\|+\beta\left\|\frac{1}{2}\left(\left|w_{n}\right|+w_{n}\right)-u\right\|,
\end{aligned}
$$

and hence $d(x, F(u))=0$. This implies that $x \in F(u)$. Similarly, we have $y \in G(u)$ and $z \in Q(y)$. This completes the proof.

From Theorem 4.1, we get the following theorem.

TheOREM 4.2. Suppose that $f, g: \mathbb{R}^{n} \rightarrow \mathbb{R}^{n}$ are Lipschitz continuous with Lipschitz constants $\delta$ and $\xi$, respectively, and $F, G: \mathbb{R}^{n} \rightarrow \mathrm{CB}\left(\mathbb{R}^{n}\right)$ are $H$-Lipschitz continuous with $H$-Lipschitz constants $\beta$ and $\eta$, respectively, and $F$ is strongly monotone with respect to $f$ with strongly monotone constant $\alpha$. If

$$
0<\rho<\frac{4(\alpha-\xi \eta)}{(\delta \beta)^{2}-(\xi \eta)^{2}}, \quad \rho \xi \eta<2, \quad \xi \eta<\min \{\alpha, \delta \beta\},
$$

then there exist $u, x, y \in \mathbb{R}^{n}$ which solve problem (2.5). Furthermore, it follows that

$$
\frac{1}{2}\left(\left|w_{n}\right|+w_{n}\right) \rightarrow u, \quad x_{n} \rightarrow x, \quad y_{n} \rightarrow y \quad \text { as } n \rightarrow \infty,
$$

where $\left\{w_{n}\right\},\left\{x_{n}\right\}$, and $\left\{y_{n}\right\}$ in $\mathbb{R}^{n}$ are the sequences generated by Algorithm 3.2.

THEOREM 4.3 [4]. Let $f, g$, and $G$ be the same as in Theorem 4.2. Further, suppose that $f$ is strongly monotone with strongly monotone constant $\alpha$. If

$$
0<\rho<\frac{4(\alpha-\xi \eta)}{\delta^{2}-(\xi \eta)^{2}}, \quad \rho \xi \eta<2, \quad \xi \eta<\alpha,
$$

then there exist $u, y \in \mathbb{R}^{n}$ which solve problem (2.6), and

$$
\frac{1}{2}\left(\left|w_{n}\right|+w_{n}\right) \longrightarrow u, \quad y_{n} \longrightarrow y, \quad \text { as } n \rightarrow \infty,
$$

where $\left\{w_{n}\right\}$ and $\left\{y_{n}\right\}$ are two sequences generated by Algorithm 3.3.

THEOREM 4.4 [14]. Let $f$ and $g$ be the same as in Theorem 4.3. If

$$
0<\rho<\frac{4(\alpha-\xi)}{\delta^{2}-\xi^{2}}, \quad \rho \xi<2, \quad \xi<\alpha,
$$

then there exists $u \in \mathbb{R}^{n}$ which is a solution of problem (2.7), and $\frac{1}{2}\left(\left|w_{n}\right|+w_{n}\right) \rightarrow u$ as $n \rightarrow \infty$, where $\left\{w_{n}\right\}$ is the sequence generated by Algorithm 3.4. 
THEOREM 4.5 [15]. Suppose that $g: \mathbb{R}^{n} \rightarrow \mathbb{R}^{n}$ is strongly monotone and Lipschitz continuous with strongly monotone constant $\alpha$ and Lipschitz constant $\delta$. If $0<\rho<$ $4 \alpha / \delta^{2}$, then there exists $u \in \mathbb{R}^{n}$ which is a solution of problem (2.8), and $\frac{1}{2}\left(\left|w_{n}\right|+w_{n}\right) \rightarrow$ $u$ as $n \rightarrow \infty$, where $\left\{w_{n}\right\}$ is the sequence generated by Algorithm 3.5.

ACKNOWLEDGEMENT. The second author was supported by the academic research fund of Ministry of Education, Republic of Korea, 1997, Project No. BSRI-97-1405.

\section{REFERENCES}

[1] S. S. Chang, Variational Inequality and Compltementarity Problem Theory with Applications, Shanghai Scientific and Tech. Literature Publishing House, Shanghai, 1991.

[2] S. S. Chang and N. J. Huang, Generalized multivalued implicit complementarity problems in Hilbert spaces, Math. Japon. 36 (1991), no. 6, 1093-1100. MR 92m:47139. Zbl 748.49006.

[3] _ Generalized strongly nonlinear quasi-complementarity problems in Hilbert spaces, J. Math. Anal. Appl. 158 (1991), no. 1, 194-202. MR 92d:90104. Zbl 739.90067.

[4] _ Generalized complementarity problems for fuzzy mappings, Fuzzy Sets and Systems 55 (1993), no. 2, 227-234. MR 94e:65067. Zbl 790.90076.

[5] R. W. Cottle, Complementarity and variational problems, Symposia Mathematica, 19 (London), Academic Press, 1976, pp. 177-208. MR 58 20486. Zbl 349.90083.

[6] R. W. Cottle and G. B. Dantzig, Complementary pivot theory of mathematical programming, Linear Algebra Appl. 1 (1968), no. 1, 103-125. MR 37\#2515. Zbl 155.28403.

[7] R. W. Cottle, J. S. Pang, and R. E. Stone, The Linear Complementarity Problem, Computer Science and Scientific Computing, Academic Press, Inc., Boston, London, 1992. MR 93f:90001. Zbl 757.90078.

[8] S. C. Fang, An iterative method for generalized complementarity problems, IEEE Trans. Automat. Control 25 (1980), no. 6, 1225-1227. MR 82i:90116. Zbl 483.49027.

[9] G. Isac, Complementarity Problems, Lecture Notes in Mathematics, vol. 1528, SpringerVerlag, Berlin, 1992. MR 94h:49002. Zbl 795.90072.

[10] S. Karamardian, Generalized complementarity problem, J. Optim. Theory Appl. 8 (1971), 161-168. MR 47 10073. Zbl 218.90052.

[11] C. E. Lemke, Bimatrix equilibrium points and mathematical programming, Management Sci. 11 (1964/1965), 681-689. MR 32\#7243. Zbl 139.13103.

[12] S. B. Nadler, Jr., Multi-valued contraction mappings, Pacific J. Math. 30 (1969), 475-488. MR 40\#8035. Zbl 187.45002.

[13] M. A. Noor, Generalized nonlinear complementarity problem, J. Natur. Sci. Math. 26 (1986), no. 1, 9-19. MR 87k:90265. Zbl 595.90088.

[14] _ Fixed point approach for complementarity problems, J. Math. Anal. Appl. 133 (1988), no. 2, 437-448. MR 89g:90223. Zbl 649.65037.

[15] M. A. Noor and S. Zarae, An iterative scheme for complementarity problems, Engrg. Anal. J. 3 (1986), 240-243.

Huang: Department of Mathematics, Sichuan University, Chengdu, Sichuan 610064, CHINA

Cho: Department of Mathematics, Gyeongsang NATIONAl University, ChinJu 660-701, KOREA 


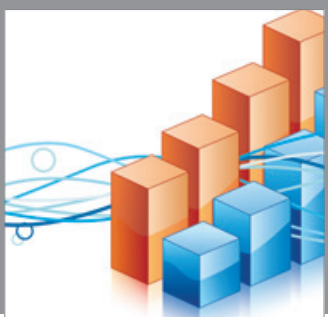

Advances in

Operations Research

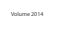

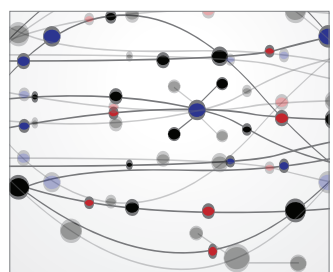

\section{The Scientific} World Journal
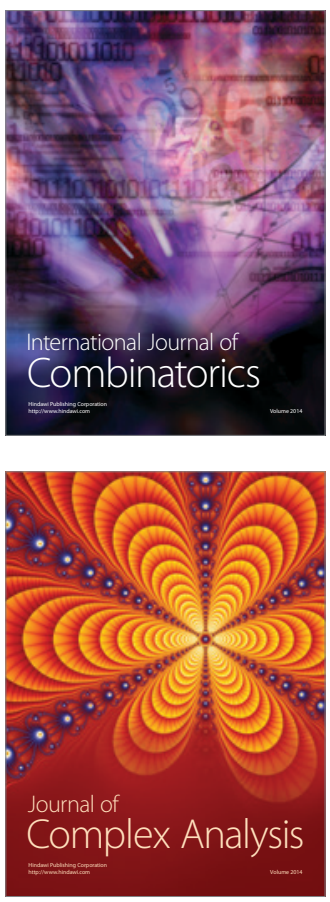

International Journal of

Mathematics and

Mathematical

Sciences
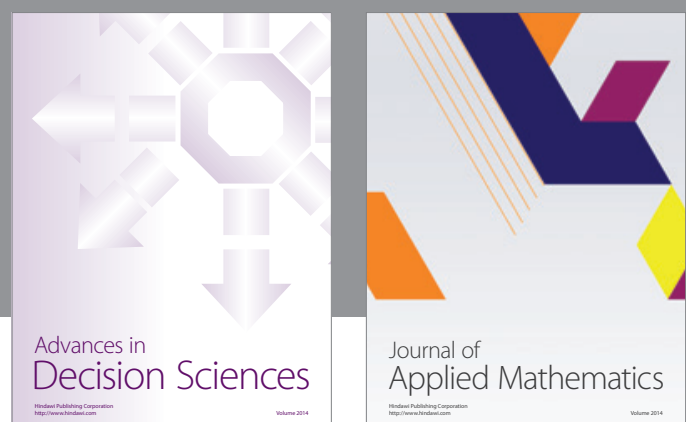

Journal of

Applied Mathematics
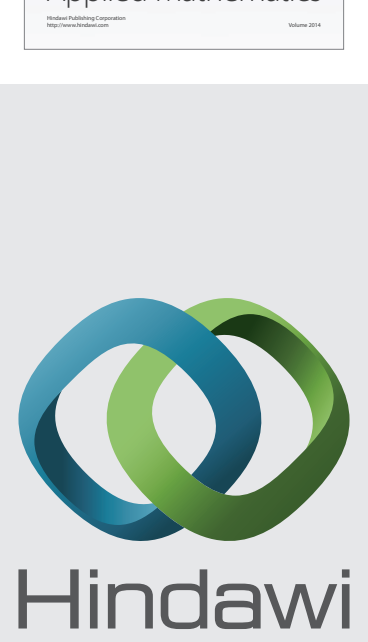

Submit your manuscripts at http://www.hindawi.com
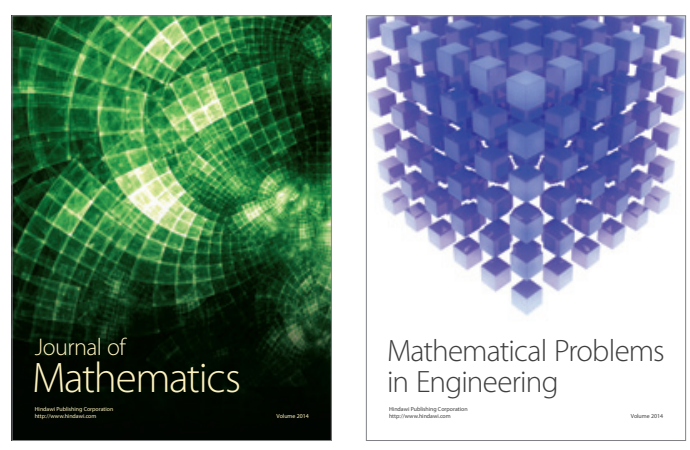

Mathematical Problems in Engineering
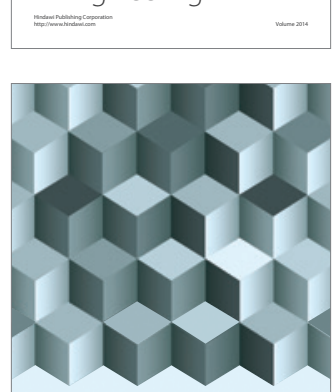

Journal of

Function Spaces
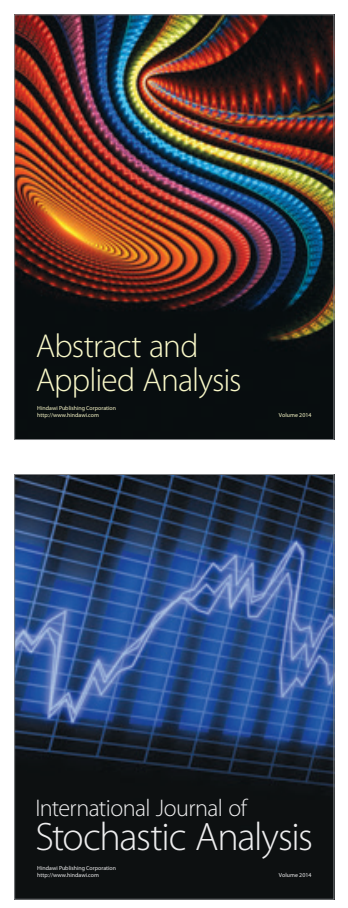

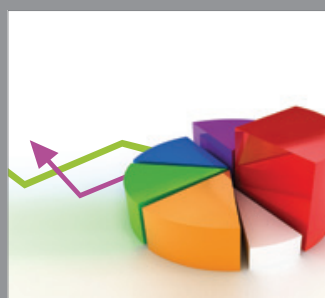

ournal of

Probability and Statistics

Promensencen
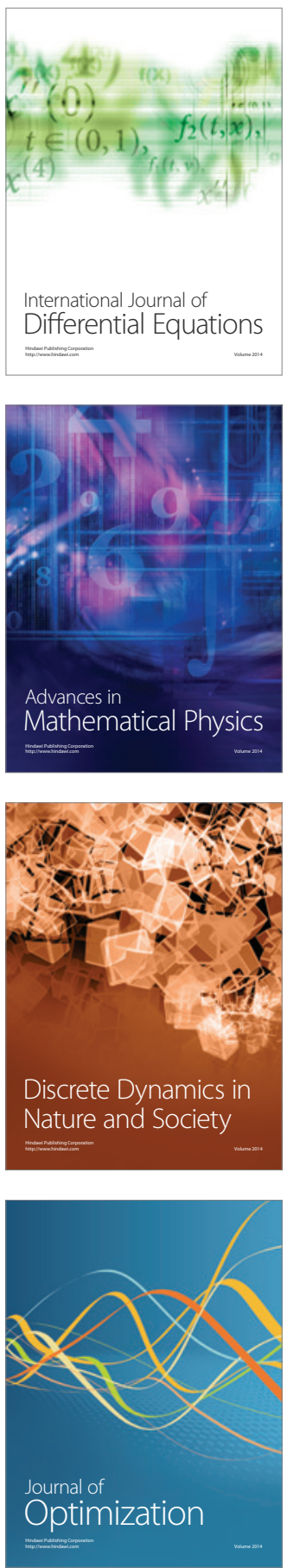